\title{
Correlation between erythropoietin receptor(s) and estrogen and progesterone receptor expression in different breast cancer cell lines
}

\author{
NINA TROŠT, NELI HEVIR, TEA LANIŠNIK RIŽNER and NATAŠA DEBELJAK \\ Institute of Biochemistry, Faculty of Medicine, University of Ljubljana, Ljubljana, Slovenia
}

Received October 9, 2012; Accepted November 29, 2012

DOI: $10.3892 /$ ijmm.2013.1231

\begin{abstract}
Erythropoietin (EPO) receptor (EPOR) expression in breast cancer has been shown to correlate with the expression of estrogen receptor (ESR) and progesterone receptor (PGR) and to be associated with the response to tamoxifen in $\mathrm{ESR}^{+} /$ $\mathrm{PGR}^{+}$tumors but not in ESR- tumors. In addition, the correlation between EPOR and $G$ protein-coupled estrogen receptor 1 [GPER; also known as G protein-coupled receptor 30 (GPR30)] has been reported, suggesting the prognostic potential of EPOR expression. Moreover, the involvement of colony stimulating factor 2 receptor, $\beta$, low-affinity (CSF2RB) and ephrin type-B receptor 4 (EPHB4) as EPOR potential receptor partners in cancer has been indicated. This study analyzed the correlation between the expression of genes for $E P O, E P O R, C S F 2 R B$, EPHB4, ESR, PGR and GPER in the MCF-7, MDA-MB-361, T-47D, MDA-MB-231, Hs578Bst, SKBR3, MCF-10A and Hs578T cell lines. The cell lines were also treated with recombinant human EPO (rHuEPO) in order to determine its ability to activate the Jak/STAT5, MAPK and PI3K signaling pathways and modify cell growth characteristics. Expression analysis stratified the cell lines in 2 main clusters, hormone-dependent cell lines expressing ESR and PGR and a hormone-independent cluster. A significant correlation was observed between the expression levels of $E S R$ and $P G R$ and their expression was also associated with that of GPER. Furthermore, the expression of GPER was associated with that of EPOR, suggesting the connection between this orphan $\mathrm{G}$ protein and EPO signaling. A negative correlation between $E P O R$ and $C S F 2 R B$ expression was observed, questioning the involvement of these two receptors in the hetero-receptor formation. rHuEPO treatment only influenced the hormone-independent cell lines, since only the MDA-MB-231, SKBR3 and Hs578T cells responded to the treatment. The correlation between the expression of the
\end{abstract}

Correspondence to: Dr Nataša Debeljak, Institute of Biochemistry, Faculty of Medicine, University of Ljubljana, Vrazov trg 2, SI-1000 Ljubljana, Slovenia

E-mail: natasa.debeljak@mf.uni-lj.si

Key words: erythropoietin receptor, ephrin type-B receptor 4, $\beta$-common receptor, estrogen receptor, progesterone receptor, $\mathrm{G}$ protein-coupled estrogen receptor 1, expression correlation, breast cancer cell lines analyzed receptors suggests that the receptors may interact in order to activate signaling pathways or to evade their inhibition. Therefore, breast cancer classification upon ESR, PGR and human epidermal growth factor receptor 2 (HER2) may not be sufficient for the selection of suitable treatment protocol. The expression of EPOR, GPER and EPHB4 may be considered as additional classification factors.

\section{Introduction}

Erythropoietin (EPO) is the core regulator of red blood cell production (1). The binding of EPO to functional erythropoietin (EPO) receptor (EPOR) homodimer triggers several downstream signaling pathways, such as the Jak2/signal transducer and activator of transcription 5 (STAT5), phosphatidylinositol 3-kinase (PI3K)/protein kinase B (Akt), Ras/mitogen-activated protein kinase (MAPK) and protein kinase $C$ pathways (2), resulting in the proliferation and differentiation of erythroid progenitors. Apart from erythropoietic cells, EPOR is expressed in many non-hematopoietic tissues, as well as in cancer (3-5). The expression of EPO and functional EPOR has also been confirmed in breast cancer tissue and cell lines $(6,7)$, the most common type of cancer among females.

Recombinant human EPO (rHuEPO) is used to correct anemia in chronic kidney disease (CKD) patients (8), chemotherapy-induced anemia (9), as well as other types of anemia. A number of pre-clinical and clinical trials have verified the use of rHuEPO as a tissue protective agent in the brain, heart and kidneys (10-12). The protective effect of EPO has been proposed to be mediated through the EPOR hetero-receptor, presumably formed with the common $\beta$ subunit of the IL-3 receptor [colony stimulating factor 2 receptor, $\beta$, low-affinity (CSF2RB)] (13). The significance of receptor tyrosine kinase ephrin type-B receptor 4 (EPHB4) in cancer development has also been indicated (14), having the potential to be another EPOR receptor partner (15). EPHB4 has been shown to be expressed in breast cancer (16). The effect of rHuEPO supportive care in cancer patients with EPOR-positive cancer is not yet well understood. Several clinical trials were terminated early due to rHuEPOnegative effects on recurrence-free survival (RFS) and overall survival or tumor progression $(5,17,18)$. Decreased patient survival may result from the rHuEPO effects on increased thrombotic events, the growth and survival of cancer cells or the attenuated sensitivity of cancer cells to various types of 
treatment (5). Nevertheless, the presence of EPO and EPOR in various cancer tissues and cell lines raises the issue of whether the use of rHuEPO as supportive care in cancer patients is appropriate (19).

Breast cancer cells express a variety of growth factor receptors determining the molecular classification of the disease (20). The most commonly expressed are the steroid hormone receptors, estrogen receptor (ESR), progesterone receptor (PGR) and androgen receptor (AR). Apart from the classical steroid hormone receptors, that are members of the nuclear receptor family (ESR, PGR and AR) and upon which the molecular classification is based, membrane-bound steroid receptors have been identified (mESR, mPGR and mAR). Membrane-initiated steroid signaling (MISS) has been implicated in intracellular signaling $(21,22)$ either in a non-transcriptional fashion by modifying existing proteins (e.g., phosphorylation) or by the modulation of gene expression and the production of proteins $(23,24)$. A cross-talk between membrane and nuclear steroid receptors has also been indicated which leads to the amplification of subsequent transcription originating from the nuclear receptors (25). The identity of cytoplasmic/membrane steroid receptors has been an issue of debate. Classical nuclear receptors, ER- $\alpha$ (ESR1) and the PGRs (PGRA and PGRB), have been observed at the plasma membrane of breast cancer cells. The palmitoylation of the classical receptors has been shown to be the signal for the membrane localization and all membrane-initiated signaling (26). In contrast to classical receptors, the membrane associated ESR is an atypical G protein-coupled receptor (GPCR), selectively activating discrete $\mathrm{G}$ protein $\alpha$ - and $\beta \gamma$-subunits that result in rapid signaling (27). Extra-nuclear signaling has been shown to modulate the proliferation, survival and invasion of breast cancer cells (28). The G protein-coupled estrogen receptor [GPER; also known as $\mathrm{G}$ protein-coupled receptor 30 (GPR30)] has been implicated in mediating estrogen action at the cell membrane; however, studies have suggested that GPER may not be a membrane ESR. A role for this orphan GPCR may involve collaboration with mESR, showing dependence on both proteins (29).

Studies have indicated the correlation between EPOR and estrogen and progesterone receptors in breast cancer cells and patients. The co-expression of EPO, EPOR and mAR protein in breast cancer patients has been shown to be negatively associated with disease-free and overall survival (30). In addition, patients with $\mathrm{ESR}^{+} / \mathrm{PGR}^{+}$tumors and low EPOR expression have significantly increased RFS following tamoxifen (TAM) treatment when compared to patients with high EPOR-expressing tumors. On the other hand, the significant improvement of RFS was detected in non-treated patients with $\mathrm{ESR}^{+}$tumors with high EPOR levels (31). Volgger et al (32) also confirmed the positive association between EPOR expression, ESR/PGR status and decreased local cancer recurrence. These observations suggest the existence of the active interplay between EPO signaling and the steroid receptors which may/can furthermore interact with other cytokine/growth factors, such as epidermal growth factor receptor (EGFR) and insulin-like growth factor 1 (IGF-I) receptor (33-36). The activation of these receptors eventually culminates in the activation of MAPK and PI3K signaling pathways which are also indicative for the receptor tyrosine kinase EPHB4 (37). The complexity of the intracellular cross-talk therefore makes it difficult to decipher the role of the particular receptor and the significance of a particular correlation.

Therefore, we aimed to assess the applicability of breast cancer cell lines in the study of the EPO signaling pathway, particularly as regards the correlation between the expression of EPOR and that of other cellular receptors. Profound differences in the expression of estrogen and progesterone receptors and estrogen metabolizing enzymes in breast cancer cell lines (38) indicate that choosing the proper cell model is of great importance. The breast cancer cell lines, MCF-7, MDA-MB361, T-47D, MDA-MB-231, Hs578Bst, SKBR3, MCF-10A and Hs578T were selected for detailed expression analysis of EPOR, ESR1-2, PGR, GPER, EPHB4, CSF 2RB and EPO. The cell lines were also analyzed for the responsiveness to $\mathrm{rHuEPO}$ induction on the level of cell proliferation and the activation of EPO signaling pathways. The phosphorylation of extracellular signal-regulated kinase (ERK)/MAPK, Akt/PI3K and STAT5/ Jak/STAT proteins was also assessed.

\section{Materials and methods}

Cell cultures. The breast cancer cell lines (Table I) were maintained in cell culture at $37^{\circ} \mathrm{C}$ in a humidified $5 \%(\mathrm{v} / \mathrm{v}) \mathrm{CO}_{2}$ atmosphere. The cell lines were obtained from the American Type Culture Collection (ATCC; Manassas, VA, USA) and were cultured according to the ATCC recommendations. The receptor status for a specific cell line and the tumor type are shown in Table I. Cells were grown in basic growth medium, supplemented with $10 \%$ FBS (38). UT7/Epo cells were used as the positive control of EPO signaling in western blot analysis. Cells were kindly provided by C. Lacout (Institute of Cancerology Gustave Roussy, Villejuif, France) and were cultured in MEM Alpha medium (Sigma, St. Louis, MO, USA), supplemented with $10 \%$ FBS and $2 \mathrm{U} / \mathrm{ml} \mathrm{rHuEPO}$ (NeoRecormon; Roche, Mannheim, Germany).

\section{Gene expression analysis}

RNA isolation. Total RNA was extracted using TRI reagent (Sigma) and treated with the DNase I (Roche) according to the manufacturer's instructions. The quality of the RNA samples was determined using an Agilent bioanalyzer assuring that all RIN values were $>9.8$. Total RNA $(1 \mu \mathrm{g})$ was transcribed to cDNA using SuperScript III reverse transcriptase (Invitrogen, USA) according to the manufacturer's instructions.

Selection of normalization gene candidates. According to our previous study (38) peptidylprolyl isomerase A (PPIA) was selected as the normalization gene from the full cohort of 16 reference genes that were tested in these cell lines.

Quantitative real-time PCR ( $q P C R)$. The expression of 9 genes of interest (Table II) and the selected normalization gene was analyzed using TaqMan ${ }^{\circledR}$ PCR assays. The expression levels were determined with the exon-spanning hydrolysis probes (FAM or VIC, dye labeled) that are commercially available as Assays-on-Demand (Applied Biosystems, Bedford, MA, USA) with optimized primer and probe concentrations (Table II). Gene expression assays covering all splice variants of the specific gene were selected; for GPER 2 assays were required in order to cover all the existing splice variants. qPCR was performed on a 384-well platform using the LightCycler $^{\circledR}$ 480 Real-Time PCR System (Roche) and TaqMan ${ }^{\circledR}$ Universal 
Table I. Details of the cohort of the selected breast cancer lines as defined by American Type Culture Collection.

\begin{tabular}{|c|c|c|c|}
\hline Cell line & $\begin{array}{l}\text { Receptor } \\
\text { status }\end{array}$ & $\begin{array}{l}\text { Tissue } \\
\text { source }\end{array}$ & $\begin{array}{l}\text { Tumor } \\
\text { type }\end{array}$ \\
\hline MCF-7 & $\mathrm{ESR}^{+}, \mathrm{PGR}^{+}$ & $\mathrm{PE}$ & IDC \\
\hline MDA-MB-361 & $\mathrm{ESR}^{+}, \mathrm{PGR}^{-}$ & $\mathrm{B}$ & $\mathrm{AC}$ \\
\hline T-47D & $\mathrm{ESR}^{+}, \mathrm{PGR}^{+}$ & PE & IDC \\
\hline MDA-MB-231 & $\mathrm{ESR}^{+}, \mathrm{PGR}^{-}$ & $\mathrm{PE}$ & $\mathrm{AC}$ \\
\hline Hs578Bst & $\mathrm{ESR}^{-}, \mathrm{PGR}^{-}$ & $\begin{array}{l}\text { Adjacent } \\
\text { breast tissue }\end{array}$ & \\
\hline SKBR3 & ESR ${ }^{-}, \mathrm{PGR}^{-}$ & PE & $\mathrm{AC}$ \\
\hline MCF-10A & $\mathrm{ESR}^{-}, \mathrm{PGR}^{-}$ & & $\mathrm{F}$ \\
\hline Hs578T & ESR-, PGR- & P.Br & IDC \\
\hline \multicolumn{4}{|c|}{$\begin{array}{l}\text { PE, pleural effusion; IDC, invasive ductal carcinoma; B, brain; AC, } \\
\text { adenocarcinoma; F, fibrocystic disease; P.Br, primary breast. Cells } \\
\text { were cultured using the same medium conditions as described in the } \\
\text { study by Hevir } \text { et al (38). }\end{array}$} \\
\hline
\end{tabular}

PCR Master Mix. Universal thermocycling parameters were applied, as recommended by the manufacturer (Applied Biosystems). The amplification of specific PCR products was performed in triplicate in a total reaction mixture of $5 \mu \mathrm{l}$ containing $0.25 \mu \mathrm{l}$ of cDNA template. Minimum Information for Publication of Quantitative Real-Time PCR Experiments (MIQE) guidelines were followed in the performance and interpretation of the qPCR reactions (39).

Western blot analysis. The expression of ERK, Akt and STAT5 proteins and their phosphorylated forms was determined by western blot analysis in the cell lysates following rHuEPO treatment. Cells were seeded on 12-well plates at the concentration of $1 \times 10^{4}$ cells/well and left in culture until they reached a confluence of $70 \%$. Prior to treatment $(24 \mathrm{~h})$ the cells were switched to serum-free medium and then treated with 5 or $25 \mathrm{U} / \mathrm{ml} \mathrm{rHuEPO}$ for 5 and $10 \mathrm{~min}$. The culture medium was then aspirated and samples were fast-frozen in liquid nitrogen.

Cell samples were lysed for $10 \mathrm{~min}$ on ice in lysis buffer as described by Kutuk et al (40) and soluble proteins were recovered in the supernatant following a 10-min centrifugation $(12,000 \mathrm{rpm})$. Samples of UT7/Epo cells treated with $2 \mathrm{U} / \mathrm{ml}$ rHuEPO were used as the positive controls. Equal amounts of protein $(50 \mu \mathrm{g})$ from each sample were loaded per well. After SDS electrophoresis, proteins were transferred onto polyvinylidene difluoride (PVDF) membranes (Immobilon-P; Millipore, Billerica, MA, USA). Membranes were blocked in a blocking solution (5\% BSA in $1 \mathrm{mM}$ PBS, $0.2 \%$ Tween-20) for $1 \mathrm{~h}$ and incubated in one of the following antibodies and dilutions: anti-ERK (1:1,000, no. 9102), anti-Akt (1:600, no. 9272), anti-STAT5 (1:600, no. 9363), anti-P-ERK (1:1,000, no. 9101), anti-P-Akt (1:600, no. 9271) and anti-P-STAT5 (1:600, no. 9351). All antibodies were purchased from Cell Signaling Technology (Danvers, MA, USA) and were raised against synthetic peptides in rabbits. As a secondary antibody,
Table II. Details of genes of interest and reference genes.

Gene

symbol Assay ID Gene name

Genes of interest

ESR1 Hs00174860_m1 Estrogen receptor 1 (ESR- $\alpha)$

ESR2 Hs00230957_m1 Estrogen receptor 2 (ESR- $\beta$ )

$P G R \quad H s 00172183 \_\mathrm{m} 1 \quad$ Progesterone receptor

EPOR Hs00959427_m1 Erythropoietin receptor

EPO Hs01071097_m1 Erythropoietin

GPER1 Hs00173506_m1 G protein-coupled estrogen receptor 1

GPER2 Hs01116133_m1 G protein-coupled estrogen receptor 2

EPHB4 Hs00174752_m1 Ephrin type-B receptor 4

$C S F 2 R B \quad H s 00166144 \_\mathrm{m} 1$ Colony stimulating factor 2 receptor, $\beta$, low-affinity (granulocyte-macrophage)

Reference gene

PPIA Hs99999904_m1 Peptidylprolyl isomerase A (cyclophilin A)

Assay ID, Assays-on-Demand (Applied Biosystems).

peroxidase-conjugated anti-rabbit IgG (1:5,000, A0545; Sigma) was used and visualized with chemiluminescence reagent (Pierce ECL Western Blotting Substrate; Thermo Scientific, Rockford, IL, USA) with a CCD camera (Fujifilm, Tokyo, Japan). Membranes were densitometrically analyzed using ImageJ software (National Institutes of Health, Bethesda, MD, USA) (41) and ratios between phosphorylated proteins to their non-phosphorylated forms were calculated and compared between samples. Experiments were repeated 3 times.

Proliferation assays. The proliferation of the MCF-7, MDA-MB-231, SKBR-3 and Hs578T cells was determined using MTT reagent (Sigma). Cells were seeded on a 96-well plate in 5-plicates at the concentration of 750 cells/well and left to adhere in the medium. Basic growth medium supplemented with 10 or $1 \%$ of FBS was used. Following a day in culture, the cells were exposed to $5 \mathrm{U} / \mathrm{ml} \mathrm{rHuEPO}$; the control cells were grown in medium without rHuEPO. Cells were maintained in culture up to 7 days and cell proliferation was measured every day. Fold change was calculated by normalizing the proliferation results with the proliferation measured at day 1. Experiments were repeated 6 times.

Statistical analysis. For correlation analysis, Pearson's correlation coefficient was defined with SPSS 19 software (SPSS Inc., Chicago, IL) using two-tailed analysis. Only correlations with a significance level of $\mathrm{P}<0.05$ were considered statistically significant. Between-group linkage was determined using hierarchical clustering analysis on an interval scale, considering Euclidean distance. The effect of rHuEPO treatment on cell proliferation was assessed by two-way analysis 
Table III. Pearson's correlation coefficients (rs) calculated for the expression of cellular receptors.

\begin{tabular}{|c|c|c|c|c|c|c|c|c|}
\hline Receptor & EPOR & $E P H B 4$ & $C S F 2 R B$ & ESR1 & ESR2 & $P G R$ & GPERI & GPER2 \\
\hline \multicolumn{9}{|l|}{ EPOR } \\
\hline $\mathrm{r}$ & 1 & $0.385^{\mathrm{c}}$ & $-0.708^{b}$ & 0.275 & 0.276 & -0.404 & $0.549^{b}$ & $0.426^{\mathrm{a}}$ \\
\hline P-value & & 0.064 & 0.003 & 0.194 & 0.268 & 0.193 & 0.006 & 0.038 \\
\hline $\mathrm{N}$ & 24 & 24 & 15 & 24 & 18 & 12 & 24 & 24 \\
\hline \multicolumn{9}{|l|}{ EPHB4 } \\
\hline $\mathrm{r}$ & & 1 & $-0.541^{\mathrm{a}}$ & 0.014 & -0.144 & $-0.871^{b}$ & $0.468^{\mathrm{a}}$ & 0.285 \\
\hline P-value & & & 0.037 & 0.949 & 0.569 & 0.000 & 0.021 & 0.177 \\
\hline $\mathrm{N}$ & & 24 & 15 & 24 & 18 & 12 & 24 & 24 \\
\hline \multicolumn{9}{|l|}{$C S F 2 R B$} \\
\hline $\mathrm{r}$ & & & 1 & -0.277 & $-0.742^{b}$ & -0.465 & -0.195 & -0.039 \\
\hline P-value & & & & 0.317 & 0.006 & 0.352 & 0.486 & 0.891 \\
\hline $\mathrm{N}$ & & & 15 & 15 & 12 & 6 & 15 & 15 \\
\hline \multicolumn{9}{|l|}{ ESRI } \\
\hline $\mathrm{r}$ & & & & 1 & 0.301 & $0.754^{\mathrm{b}}$ & 0.361 & $0.443^{\mathrm{a}}$ \\
\hline P-value & & & & & 0.224 & 0.005 & 0.083 & 0.030 \\
\hline $\mathrm{N}$ & & & & 24 & 18 & 12 & 24 & 24 \\
\hline \multicolumn{9}{|l|}{ ESR2 } \\
\hline $\mathrm{r}$ & & & & & 1 & $0.945^{\mathrm{b}}$ & -0.356 & $-0.499^{a}$ \\
\hline P-value & & & & & & 0.000 & 0.148 & 0.035 \\
\hline $\mathrm{N}$ & & & & & 18 & 12 & 18 & 18 \\
\hline \multicolumn{9}{|l|}{$P G R$} \\
\hline $\mathrm{r}$ & & & & & & 1 & $-0.539^{c}$ & -0.368 \\
\hline P-value & & & & & & & 0.071 & 0.239 \\
\hline $\mathrm{N}$ & & & & & & 12 & 12 & 12 \\
\hline \multicolumn{9}{|l|}{ GPERI } \\
\hline $\mathrm{r}$ & & & & & & & 1 & $0.884^{\mathrm{b}}$ \\
\hline P-value & & & & & & & & 0.000 \\
\hline $\mathrm{N}$ & & & & & & & 24 & 24 \\
\hline \multicolumn{9}{|l|}{ GPER2 } \\
\hline $\mathrm{r}$ & & & & & & & & 1 \\
\hline \multicolumn{9}{|l|}{ P-value } \\
\hline $\mathrm{N}$ & & & & & & & & 24 \\
\hline
\end{tabular}

Sample number $(\mathrm{N})$ denotes the number of samples upon which $r$ was calculated. ${ }^{\text {a }}$ Statistical significance for Type 1 error $\alpha=0.05 .{ }^{b}$ Statistical significance for Type 1 error $\alpha=0.01 .{ }^{c}$ Marginal statistical significance for Type 1 error $0.05<\alpha<0.08$.

of variance (ANOVA). $\mathrm{P}<0.05$ was considered to indicate a statistically significant difference.

\section{Results}

Expression of EPO, EPOR and its potential receptor partners. EPO expression was confirmed in the SKBR3 cell line (Fig. 1) while all the other cell lines were negative for the expression of this gene. EPOR and EPHB4 were expressed in all of the examined breast cancer cell lines with $E P H B 4$ expression levels being substantially higher compared to those of EPOR. On the other hand, $C S F 2 R B$ was only weakly expressed in some of the examined cell lines (Fig. 1).
Expression of genes for estrogen and progesterone receptors. The expression of genes for ESR1, ESR2 and PGR is comparable with previously published data (38), confirming the hormone dependency of the MCF-7, MDA-MB-361 and T-4TD cell lines. In addition, the MCF-7 and MDA-MB-361 cell lines showed a high expression of GPER, while T-47D cells were negative for the expression of this gene. Low levels of both GPER isoforms were confirmed in the Hs578Bst, SKBR3, MCF-10A and Hs578T cell lines (Fig. 1).

Correlation analysis. A variety of correlations in the expression of the analyzed genes were confirmed for the cohort of the analyzed cell lines (Table III). 

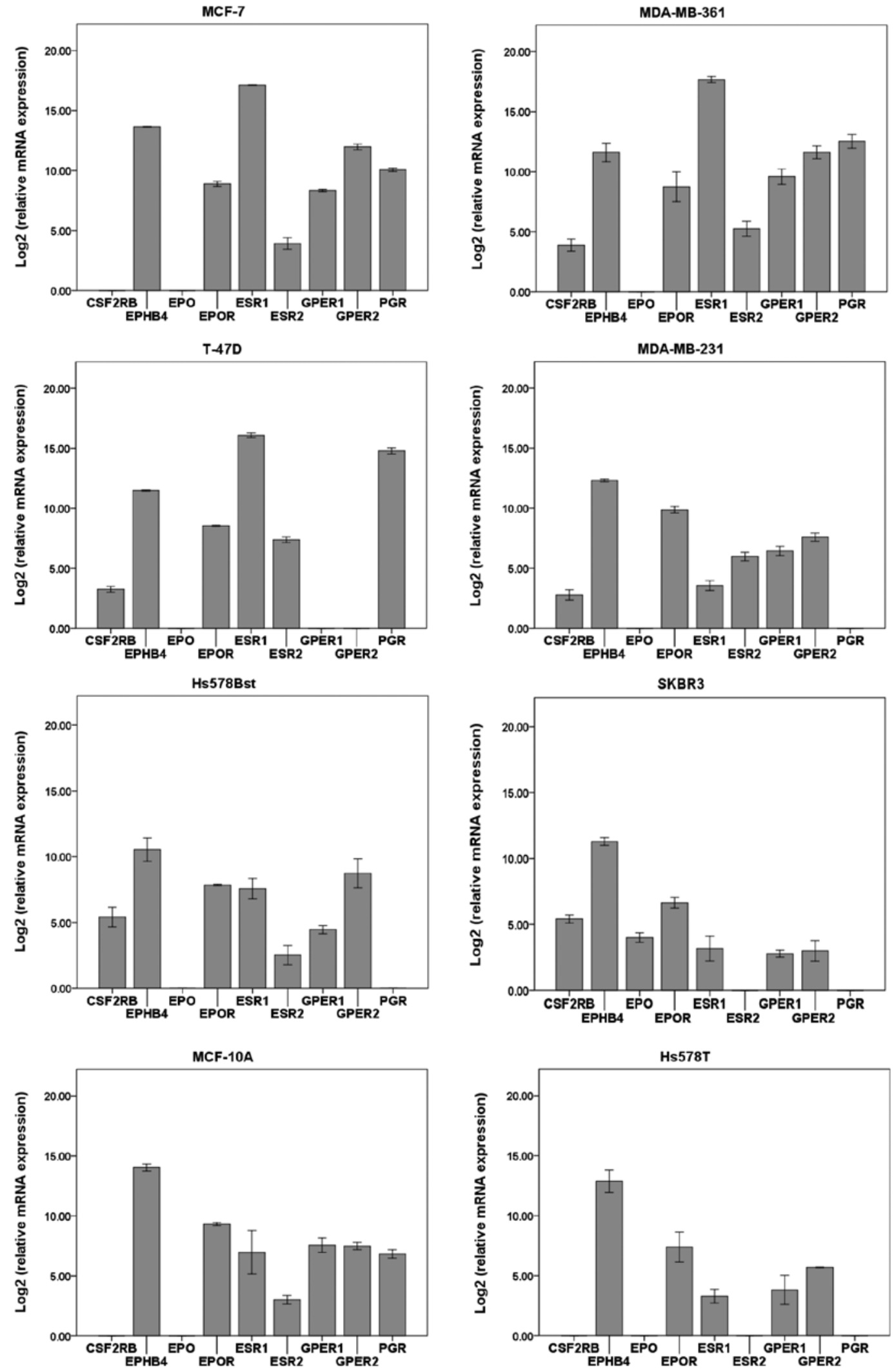

Figure 1. Expression of EPOR, EPHB4, CSF2RB, ESR1, ESR2, PGR, GPER1, GPER2 and EPO in our cohort of breast cancer cell lines. Error bars represent standard deviation between sample triplicates.

Hierarchical clustering. Based on the gene expression data, the cell lines were clustered in 5 subgroups (Fig. 2). Principally, the cell lines were stratified in 2 main clusters: a cluster of hormone-dependent breast cancer cell lines expressing ESR and PGR (Fig. 2, grey box) and a cluster of hormoneindependent breast cancer cell lines (Fig. 2, white box). 


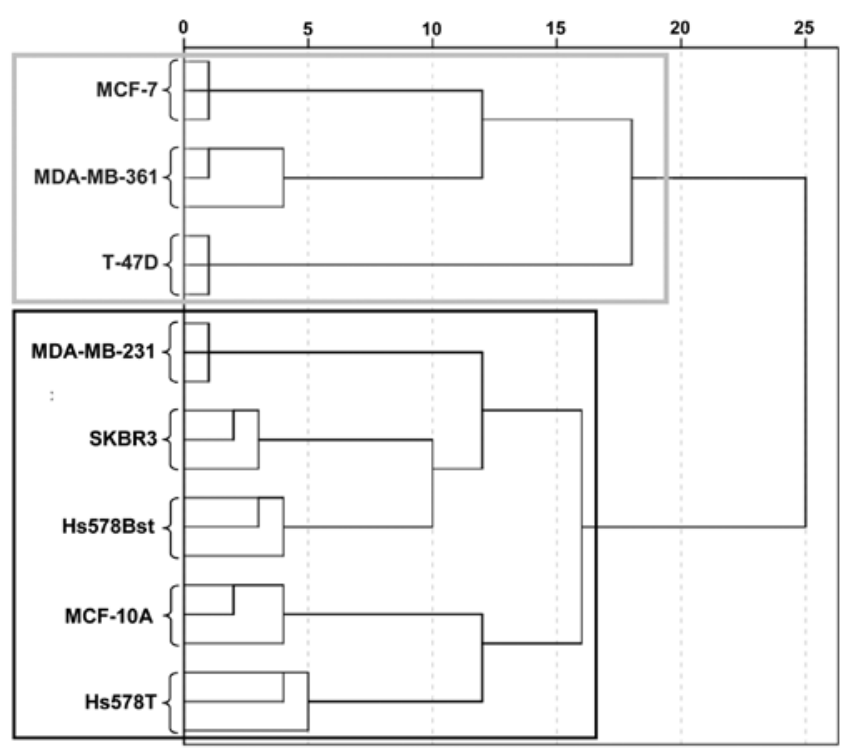

Hormone-dependent breast cancer cell lines Hormone-independent breast cancer cell lines

Figure 2. Hierarchical clustering upon qPCR results. Two main clusters forms: gray, hormone-dependent breast cancer cell lines; white, hormoneindependent breast cancer cell lines. An individual cell line was represented with 3 samples. Expression results of all 4 samples were included in a clustering analysis.

\section{Cell responsiveness to $\mathrm{rH} \mathrm{HPO}$ induction}

EPO involvement in the activation of the MAPK, PI3K and STAT5 signaling pathways. EPO has been shown to promote the activation of the MAPK, PI3K and Jak/STAT signaling pathways $(2,42)$. Therefore, we evaluated whether rHuEPO treatment promotes the phosphorylation of ERK (MAPK), Akt (PI3K) and STAT5 (Jak/STAT) proteins in our breast cancer cell lines. The MDA-MB-231, SKBR3 and Hs578T cells were the only ones showing some responsiveness to $\mathrm{rHuEPO}$ treatment (Fig. 3), which was more evident 10 min after rHuEPO induction. A slight increase in phosphorylated ERK and Akt was detected in the rHuEPO-treated SKBR3 and MDA-MB-231 cells compared to the controls. An increased Akt phosphorylation in SKBR3 cells was evident only when using $25 \mathrm{U} / \mathrm{ml} \mathrm{rHuEPO}$, otherwise it was independent of the concentration used (Fig. 3). STAT5 phosphorylation seems to be cell-specific since it was confirmed only in the SKBR3 cells (Fig. 3); its phosphorylation was slightly increased $10 \mathrm{~min}$ after rHuEPO induction. In the MCF-10A cells, Akt phosphorylation could not be confirmed at defined experimental conditions. The Hs578T cells responded to $\mathrm{rHuEPO}$ with a decreased level of phosphorylated ERK and Akt at both indicated time-points; the decrease was more pronounced with $5 \mathrm{U} / \mathrm{ml} \mathrm{rHuEPO.}$

Cell proliferation. Based on the clustering results, the MCF-7, MDA-MB-231, SKBR3 and Hs578T cell lines were chosen and analyzed for their responsiveness to $\mathrm{rHuEPO}$ induction in terms of cell proliferation. We could not confirm any rHuEPO effect on MCF-7 and MDA-MB-231 cell proliferation under our experimental conditions (data not shown). However, the rHuEPO treated Hs578T $(\mathrm{p}<0.001)$ and SKBR3 cells $(p=0.014)$ showed a significant decrease in cell proliferation when cultivated in the medium supplemented with
1\% FBS (Fig. 4). rHuEPO did not influence Hs578T and SKBR3 cell proliferation when the cells were grown in the medium supplemented with $10 \%$ FBS (data not shown).

\section{Discussion}

Breast cancer is the most common type of cancer afflicting females in the Western world and as such represents an important health issue. Estrogens and progesterone have been shown to be implicated in the development of the disease; therefore, the determination of the ESR/PGR status is used as a strong predictive factor. In addition, the EPOR/ESR/ PGR status has been suggested to be of prognostic value for the therapeutic response and RFS (31). The latest insights into the pathology of breast cancer has revealed the interaction between nuclear and membrane ESR, connecting these receptors with the EGFR and GPCR receptor families (25). Breast cancer cell lines (Table I) were therefore analyzed in detail for the gene expression of $E P O, E P O R$, potential EPOR receptor partners (EPHB4 and CSF $2 R B)$, several intracellular steroid receptors (ESRI-2 and $P G R$ ) and GPER and the correlation between the expression of these genes was determined.

The expression of EPOR was confirmed in all cell lines in accordance with previous data (6), as was the expression of EPHB4 (16). Furthermore, a correlation between $E P H B 4$ and EPOR was indicated, proposing its potential to be involved in the EPOR signaling pathway (Table III). On the other hand, $C S F 2 R B$ was weakly expressed only in some of the examined cell lines and negatively correlated with the expression of EPOR (Table III), thus questioning its involvement in the formation of the EPOR-CSF2RB heteroreceptor in the analyzed cell lines. The expression of $E P O$ was confirmed only in the SKBR3 cells, suggesting that in the other selected cell lines endogenous EPO does not act as an autocrine activator of EPOR and downstream signaling pathways.

The expression levels of genes for ESR (ESRl and ESR2) and $P G R$ are in accordance with the ATCC data (Table I), except for some of the analyzed cell lines (MDA-MB-361, Hs578Bst, MCF-10A and Hs578T). The ATCC data is based on the immunohistochemical protein detection while our data represent mRNA expression levels that do not necessarily coincide with the functional protein expression. The ESR receptor genes showed a strong correlation with the expression of $P G R$ and to a lesser extent also with that of GPER. A positive correlation between ESR 1 and GPER2 genes in breast cancer cell lines indicates that these proteins may interact at the plasma membrane, an observation that has previously been shown in endometrial and ovarian cancer cells and keratinocytes. In these cells, the cross-talk between membrane-bound ESR1 and GPER proteins mediates rapid EGFR-dependent kinase signaling by $17 \beta$-estradiol to $c$-FOS and cyclin D1 (CCND1) upregulation and proliferation (43-45). The correlation between GPER splice variants was confirmed as expected.

Larsson et al (31) reported the positive correlation between EPOR and ESR/PGR expression in breast cancer patients. We could not confirm this correlation in our cohort of analyzed cell lines. On the other hand, we were able to confirm the positive correlation between EPOR and GPER which is in 

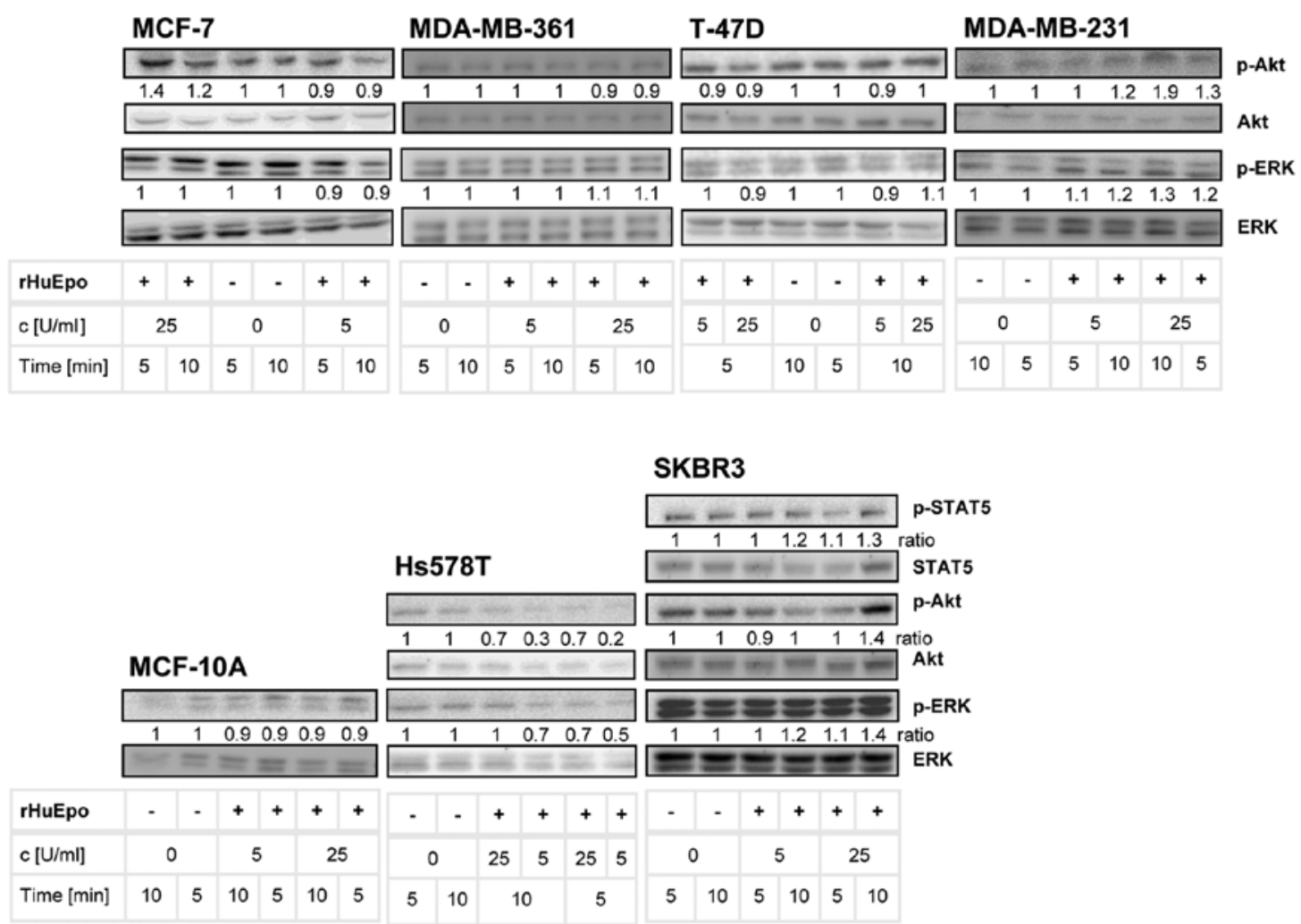

Figure 3. Involvement of MAPK (ERK), PI3K (Akt) and Jak/STAT (STAT5) signaling pathways in EPO signaling. The ratios represent quantitative analysis of densitometric values of specific band intensities normalized to the value of the corresponding untreated controls, which was arbitrarily set to 1 .
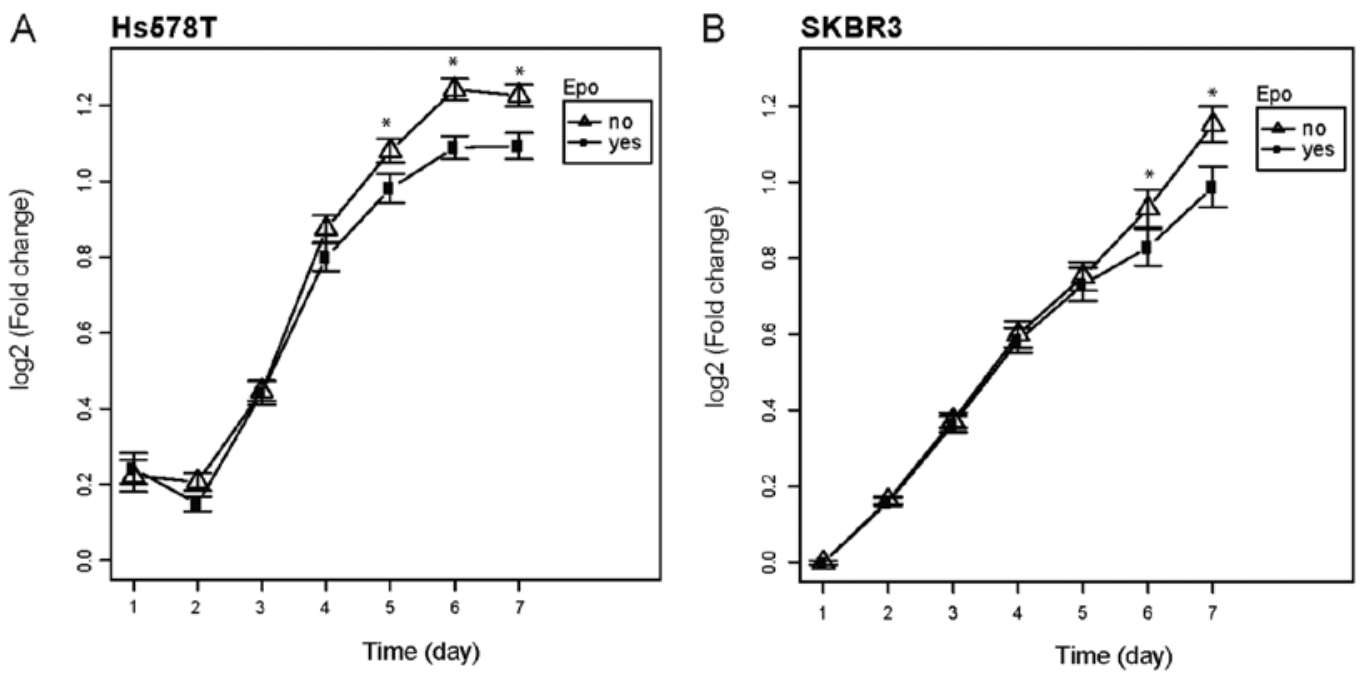

Figure 4. rHuEPO effect on cell proliferation in (A) the Hs578T ( $<<0.001)$ and (B) SKBR3 ( $=0.014)$ cell line. $\mathbf{m}$, rHuEPO-treated cells; $\triangle$, rHuEPO nontreated cells. Fold change was calculated by normalizing proliferation results with the proliferation at Day 1. Error bars represent standard deviations in fold change ratios between 3 experiments. "Statistically significant difference for Type I error $\alpha=0.05$.

agreement with previously published data, connecting GPER to EPO signaling $(30,36)$. Larsson et al (31) also reported that high EPOR expression in $\mathrm{ESR}^{+} / \mathrm{PGR}^{+}$breast cancer patients negatively affects their responses to TAM treatment. TAM has previously been reported to convert from antagonist to agonist in breast cancer, depending on the phosphorylation of ESR (46). The enhanced cross-talk between membrane ESR and EGFR family member receptors, most notably human epidermal growth factor receptor 2 (HER2), may be associated with the development of TAM resistance in breast cancer, possibly by stimulating nuclear ESR phosphorylation $(31,47,48)$. Liang et al (49) reported that EPOR signaling can circumvent the trastuzumab inhibition of HER2. The inhibition of ESR by TAM may also be hindered by the crosstalk between HER2, GPER and EPOR (48) proteins. This hypothesis needs to be further validated. 
Connecting cell response to $\mathrm{rH} E \mathrm{EPO}$ with the growth factor expression profile. The MCF-7, MDA-MB-231, SKBR3 and Hs578T cell lines were selected as representatives for a particular molecular subtype (Fig. 2) and analyzed for their responsiveness to $\mathrm{rHuEPO}$ treatment. The hormone-dependent $\mathrm{MCF}-7$ (ESR $\left.{ }^{+} / \mathrm{PGR}^{+}\right)$cells showed no significant difference in cell proliferation when exposed to rHuEPO compared to the untreated cells. This result is in agreement with the observation that rHuEPO cannot activate cell signaling in these cells (Fig. 3). Similarly, no change in cell proliferation was detected with the more invasive MDA-MB-231 (ESR $+\mathrm{PGR}^{-}$) cells, though the increase in the phosphorylation of ERK and Akt was confirmed. MDA-MB-231 cells are PGR-negative and as such represent a more aggressive breast cancer phenotype which is more independent of growth factor withdrawal (50). Furthermore, this cell line expresses a mutated form of tumor protein 53 (p53) which has previously been implicated in the regulation of cell survival following exposure to EPO and cisplatin (51) and serum deprivation (52). On the other hand, Hs578T (ESR-/PGR') and SKBR3 (ESR-/PGR-) cells showed decreased cell proliferation when treated with $\mathrm{rHuEPO}$ in a medium containing a decreased FBS concentration. Different FBS concentrations were used on account of a possible mitogenic synergism between $\mathrm{rHuEPO}$ and serum-contained growth factors or cytokines. The decrease in proliferation was more evident in the Hs578T cells which also showed decreased levels of ERK and Akt phosphorylation following rHuEPO induction. The decrease in SKBR3 cell proliferation was surprising, seeing that the 3 EPO-indicative signaling pathways were activated upon rHuEPO treatment. The mechanism of action in the SKBR3 cell line has to be further analyzed.

In conclusion, the molecular classification of breast cancer is based upon the expression of ESR, PGR and HER2; however, our results indicate that intracellular receptors may cross-talk in order to activate signaling pathways or evade their inhibition. Our current analysis on breast cancer cell lines confirmed the proposed correlation between GPER and other cellular receptors, as well as EPOR, characterizing them as a suitable model for detailed mechanistic analysis. Further analysis of ESR, PGR, EPOR, EPHB4 and GPER interaction on the protein level needs to be carried out. The results from the present study suggest that the expression of EPOR, membrane receptor GPER and EPHB4 may be considered as an additional classification factor before choosing a suitable treatment protocol.

\section{Acknowledgements}

Authors thank Dr Peter Juvan for assistance with statistical analysis. Dr Toni Petan and Dr Igor Križaj are acknowledged for providing the cell lines. This study was supported by the J3-0124 grant to N.D., J3-4135 grant to T.L.R. and Young Researcher grants to N.T. and N.H., all from the Slovenian Research Agency (ARRS).

\section{References}

1. Sytkowski JA: Erythropoietin: Blood, Brain and Beyond. WileyVCH, Boston, MA, 2004.
2. Debeljak N and Sytkowski AJ: EpoR. UCSD-Nature Molecule Pages, 2007. (doi: 10.1038/mp.a000863.01).

3. Jelkmann W and Wagner K: Beneficial and ominous aspects of the pleiotropic action of erythropoietin. Ann Hematol 83: 673-686, 2004.

4. Debeljak N and Sytkowski AJ: Erythropoietin: new approaches to improved molecular designs and therapeutic alternatives. Curr Pharm Des 14: 1302-1310, 2008.

5. Szenajch J, Wcislo G, Jeong JY, Szczylik C and Feldman L: The role of erythropoietin and its receptor in growth, survival and therapeutic response of human tumor cells: from clinic to bench - a critical review. Biochim Biophys Acta 1806: 82-95, 2010.

6. Acs G, Acs P, Beckwith SM, et al: Erythropoietin and erythropoietin receptor expression in human cancer. Cancer Res 61: 3561-3565, 2001.

7. Arcasoy MO, Amin K, Karayal AF, et al: Functional significance of erythropoietin receptor expression in breast cancer. Lab Invest 82: 911-918, 2002.

8. Eschbach JW, Egrie JC, Downing MR, Browne JK and Adamson JW: Correction of the anemia of end-stage renal disease with recombinant human erythropoietin. Results of a combined phase I and II clinical trial. N Engl J Med 316: 73-78, 1987.

9. Schrijvers D, De Samblanx H and Roila F; ESMO Guidelines Working Group: Erythropoiesis-stimulating agents in the treatment of anaemia in cancer patients: ESMO Clinical Practice Guidelines for use. Ann Oncol 21 (Suppl 5): v244-v247, 2010.

10. Latini R, Brines M and Fiordaliso F: Do non-hemopoietic effects of erythropoietin play a beneficial role in heart failure? Heart Fail Rev 13: 415-423, 2008.

11. Moore EM, Bellomo R and Nichol AD: Erythropoietin as a novel brain and kidney protective agent. Anaesth Intensive Care 39: 356-372, 2011.

12. Sytkowski AJ: The neurobiology of erythropoietin. Cell Mol Neurobiol 31: 931-937, 2011.

13. Brines M, Grasso G, Fiordaliso F, et al: Erythropoietin mediates tissue protection through an erythropoietin and common beta-subunit heteroreceptor. Proc Natl Acad Sci USA 101: 14907-14912, 2004.

14. Noren NK and Pasquale EB: Paradoxes of the EphB4 receptor in cancer. Cancer Res 67: 3994-3997, 2007.

15. Jackson DB, Stein M, Voss H, Brock S, Danes CG and Sood A: Tissue protective erythropoietin receptor (nepor) and methods to use. Patent WO 2009/068677. Filed November 28, 2008; issued April 18, 2012.

16. Kumar SR, Singh J, Xia G, et al: Receptor tyrosine kinase EphB4 is a survival factor in breast cancer. Am J Pathol 169: 279-293, 2006.

17. Henke M, Laszig R, Rube C, et al: Erythropoietin to treat head and neck cancer patients with anaemia undergoing radiotherapy: randomised, double-blind, placebo-controlled trial. Lancet 362: 1255-1260, 2003.

18. Leyland-Jones B, Semiglazov V, Pawlicki M, et al: Maintaining normal hemoglobin levels with epoetin alfa in mainly nonanemic patients with metastatic breast cancer receiving first-line chemotherapy: a survival study. J Clin Oncol 23: 5960-5972, 2005.

19. Sytkowski AJ: Does erythropoietin have a dark side? Epo signaling and cancer cells. Sci STKE 2007: pe38, 2007.

20. Schnitt SJ: Classification and prognosis of invasive breast cancer: from morphology to molecular taxonomy. Mod Pathol 23 (Suppl 2): S60-S64, 2010.

21. Thomas P, Pang Y, Filardo EJ and Dong J: Identity of an estrogen membrane receptor coupled to a $\mathrm{G}$ protein in human breast cancer cells. Endocrinology 146: 624-632, 2005.

22. Thomas P: Characteristics of membrane progestin receptor alpha (mPRalpha) and progesterone membrane receptor component 1 (PGMRC1) and their roles in mediating rapid progestin actions. Front Neuroendocrinol 29: 292-312, 2008.

23. Levin ER: Integration of the extranuclear and nuclear actions of estrogen. Mol Endocrinol 19: 1951-1959, 2005.

24. Bjornstrom L and Sjoberg M: Mechanisms of estrogen receptor signaling: convergence of genomic and nongenomic actions on target genes. Mol Endocrinol 19: 833-842, 2005.

25. Vasudevan $\mathrm{N}$ and Pfaff DW: Membrane-initiated actions of estrogens in neuroendocrinology: emerging principles. Endocr Rev 28: 1-19, 2007.

26. Pedram A, Razandi M, Sainson RC, Kim JK, Hughes CC and Levin ER: A conserved mechanism for steroid receptor translocation to the plasma membrane. J Biol Chem 282: 22278-22288, 2007. 
27. Kumar $\mathrm{P}, \mathrm{Wu} \mathrm{Q}$, Chambliss KL, et al: Direct interactions with $\mathrm{G} \alpha \mathrm{i}$ and $\mathrm{G} \beta \gamma$ mediate nongenomic signaling by estrogen receptor $\alpha$. Mol Endocrinol 21: 1370-1380, 2007.

28. Hammes SR and Levin ER: Minireview: Recent advances in extranuclear steroid receptor actions. Endocrinology 152: 4489-4495, 2011.

29. Levin ER: G protein-coupled receptor 30: estrogen receptor or collaborator? Endocrinology 150: 1563-1565, 2009.

30. Pelekanou V, Kampa M, Kafousi M, et al: Erythropoietin and its receptor in breast cancer: correlation with steroid receptors and outcome. Cancer Epidemiol Biomarkers Prev 16: 2016-2023, 2007.

31. Larsson AM, Jirstrom K, Fredlund E, et al: Erythropoietin receptor expression and correlation to tamoxifen response and prognosis in breast cancer. Clin Cancer Res 15: 5552-5559, 2009.

32. Volgger B, Kurz K, Zoschg K, et al: Importance of erythropoetin receptor expression in tumour tissue for the clinical course of breast cancer. Anticancer Res 30: 3721-3726, 2010.

33. Filardo EJ, Quinn JA and Sabo E: Association of the membrane estrogen receptor, GPR30, with breast tumor metastasis and transactivation of the epidermal growth factor receptor. Steroids 73: 870-873, 2008.

34. Kampa M, Pelekanou V and Castanas E: Membrane-initiated steroid action in breast and prostate cancer. Steroids 73: 953-960, 2008.

35. Pelekanou V, Notas G, Sanidas E, Tsapis A, Castanas E and Kampa M: Testosterone membrane-initiated action in breast cancer cells: Interaction with the androgen signaling pathway and EPOR. Mol Oncol 4: 135-149, 2010.

36. Notas G, Kampa M, Pelekanou V and Castanas E: Interplay of estrogen receptors and GPR30 for the regulation of early membrane initiated transcriptional effects: a pharmacological approach. Steroids 77: 943-950, 2011.

37. Xiao Z, Carrasco R, Kinneer K, et al: EphB4 promotes or suppresses Ras/MEK/ERK pathway in a context-dependent manner: implications for EphB4 as a cancer target. Cancer Biol Ther 13: 630-637, 2012.

38. Hevir N, Trošt N, Debeljak N and Rižner TL: Expression of estrogen and progesterone receptors and estrogen metabolizing enzymes in different breast cancer cell lines. Chem Biol Interact 191: 206-216, 2011.

39. Bustin SA, Benes V, Garson JA, et al: The MIQE guidelines: minimum information for publication of quantitative real-time PCR experiments. Clin Chem 55: 611-622, 2009.

40. Kutuk O, Arisan ED, Tezil T, Shoshan MC and Basaga $\mathrm{H}$ : Cisplatin overcomes Bcl-2-mediated resistance to apoptosis via preferential engagement of Bak: critical role of Noxa-mediated lipid peroxidation. Carcinogenesis 30: 1517-1527, 2009.
41. Abramoff MD, Magelhaes PJ and Ram SJ: Image Processing with ImageJ. Biophotonics International 11: 36-42, 2004.

42. Jelkmann W, Bohlius J, Hallek M and Sytkowski AJ: The erythropoietin receptor in normal and cancer tissues. Crit Rev Oncol Hematol 67: 39-61, 2008.

43. Vivacqua A, Bonofiglio D, Recchia AG, et al: The G proteincoupled receptor GPR30 mediates the proliferative effects induced by 17 beta-estradiol and hydroxytamoxifen in endometrial cancer cells. Mol Endocrinol 20: 631-646, 2006.

44. Albanito L, Madeo A, Lappano R, et al: G protein-coupled receptor 30 (GPR30) mediates gene expression changes and growth response to 17 beta-estradiol and selective GPR30 ligand G-1 in ovarian cancer cells. Cancer Res 67: 1859-1866, 2007.

45. Kanda $\mathrm{N}$ and Watanabe $\mathrm{S}$ : 17beta-estradiol stimulates the growth of human keratinocytes by inducing cyclin D2 expression. J Invest Dermatol 123: 319-328, 2004.

46. Michalides R, Griekspoor A, Balkenende A, et al: Tamoxifen resistance by a conformational arrest of the estrogen receptor alpha after PKA activation in breast cancer. Cancer Cell 5: 597-605, 2004

47. Shou J, Massarweh S, Osborne CK, et al: Mechanisms of tamoxifen resistance: increased estrogen receptor-HER2/neu cross-talk in ER/HER2-positive breast cancer. J Natl Cancer Inst 96: 926-935, 2004.

48. Ignatov A, Ignatov T, Roessner A, Costa SD and Kalinski T: Role of GPR30 in the mechanisms of tamoxifen resistance in breast cancer MCF-7 cells. Breast Cancer Res Treat 123: 87-96, 2010.

49. Liang K, Esteva FJ, Albarracin C, et al: Recombinant human erythropoietin antagonizes trastuzumab treatment of breast cancer cells via Jak2-mediated Src activation and PTEN inactivation. Cancer Cell 18: 423-435, 2010.

50. Cui X, Schiff R, Arpino G, Osborne CK and Lee AV: Biology of progesterone receptor loss in breast cancer and its implications for endocrine therapy. J Clin Oncol 23: 7721-7735, 2005.

51. Trošt N, Juvan P, Serša G and Debeljak N: Contrasting effect of erythropoietin on breast cancer cell response to cisplatin induced cytotoxicity. Radiol Oncol 46: 213-225, 2012.

52. Hui L, Zheng Y, Yan Y, Bargonetti J and Foster DA: Mutant p53 in MDA-MB-231 breast cancer cells is stabilized by elevated phospholipase D activity and contributes to survival signals generated by phospholipase D. Oncogene 25: 73057310,2006 